\title{
Functional Characterization of 21 Allelic Variants of Dihydropyrimidine Dehydrogenase Identified in 1070 Japanese Individuals
}

\author{
Eiji Hishinuma, Yoko Narita, Sakae Saito, Masamitsu Maekawa, Fumika Akai, Yuya Nakanishi, \\ Jun Yasuda, Masao Nagasaki, Masayuki Yamamoto, Hiroaki Yamaguchi, Nariyasu Mano, \\ Noriyasu Hirasawa, and Masahiro Hiratsuka
}

Laboratory of Pharmacotherapy of Life-Style Related Diseases, Graduate School of Pharmaceutical Sciences (E.H., Yo.N., F.A., Yu. N., N.H., M.H.), and Tohoku Medical Megabank Organization (S.S., J.Y., M.N., M.Y., M.H.), and Department of Pharmaceutical Sciences, Tohoku University Hospital, Sendai, Japan (M.M., H.Y., N.M., M.H.)

Received March 27, 2018; accepted May 11, 2018

\begin{abstract}
Dihydropyrimidine dehydrogenase (DPD, EC 1.3.1.2), encoded by the DPYD gene, is the rate-limiting enzyme in the degradation pathway of endogenous pyrimidine and fluoropyrimidine drugs such as 5-fluorouracil (5-FU). DPD catalyzes the reduction of uracil, thymine, and 5-FU. In Caucasians, DPYD mutations, including $D P Y D * 2 A, D P Y D * 13$, c.2846A $>\mathrm{T}$, and c.1129-5923C $>\mathrm{G} / \mathrm{hapB} 3$, are known to contribute to interindividual variations in the toxicity of 5-FU; however, none of these DPYD polymorphisms has been identified in the Asian population. Recently, 21 DPYD allelic variants, including some novel single-nucleotide variants (SNVs), were identified in $\mathbf{1 0 7 0}$ healthy Japanese individuals by analyzing their whole-genome sequences (WGSs), but the functional alterations caused by these variants remain unknown. In this study, in vitro analysis was performed on 22 DPD allelic variants by transiently
\end{abstract}

5-Fluorouracil (5-FU) is the anticancer drug most frequently prescribed for use in the treatment of various solid tumors, such as those found in the gastrointestinal tract, breast, head, and neck (Ide et al., 2013; Okuma et al., 2016). 5-FU has a narrow therapeutic index, and approximately $10 \%-30 \%$ of patients treated with 5-FU-based regimens develop early onset severe or life-threatening toxicity (Rothenberg et al., 2001; Twelves et al., 2005; Saltz et al., 2007). Therefore, a biomarker for the prediction of adverse effects before 5-FU administration is necessary.

More than $80 \%$ of the administered dose of 5-FU is rapidly degraded by three consecutive enzymes of the endogenous pyrimidine, uracil, and

This study was supported in part by the Foundation for Promotion of Cancer Research in Japan [Grants 159 and 100], Tohoku Medical Megabank Project from the Ministry of Education,Culture, Sports, Science and Technology (MEXT) and the Japan Agency for Medical Research and Development (AMED) [Grant JP17km0105002].

https://doi.org/10.1124/dmd.118.081737 expressing wild-type DPD and 21 DPD variants in 293FT cells and characterizing their enzymatic activities using $5-\mathrm{FU}$ as a substrate. DPD expression levels and dimeric forms were determined using immunoblotting and blue-native PAGE, respectively. Additionally, the values of three kinetic parameters-the Michaelis constant $\left(K_{m}\right)$, maximum velocity $\left(V_{\text {max }}\right)$, and intrinsic clearance $\left(C L_{i n t}=V_{\text {max }} / K_{m}\right)$-were determined for the reduction of 5-FU. Eleven variants exhibited significantly decreased intrinsic clearance compared with wild-type DPD. Moreover, the band patterns observed in the immunoblots of blue-native gels indicated that DPD dimerization is required for enzymatic activity in DPD. Thus, the detection of rare DPYD variants might facilitate severe adverse effect prediction of 5-FU-based chemotherapy in the Japanese population.

ABBREVIATIONS: $\beta$-NADPH, $\beta$-nicotinamide adenine dinucleotide phosphate; DPD, dihydropyrimidine dehydrogenase; FAD, flavin adenine dinucleotide; FMN, flavin mono nucleotide; 5-FU, 5-fluorouracil; FUH ${ }_{2}$, dihydro-5-fluorouracil; GAPDH, glyceraldehyde 3-phosphate dehydrogenase; HRP, horseradish peroxidase; SNV, single-nucleotide variant; ToMMo, Tohoku Medical Megabank Organization; WGS, wholegenome sequence. 


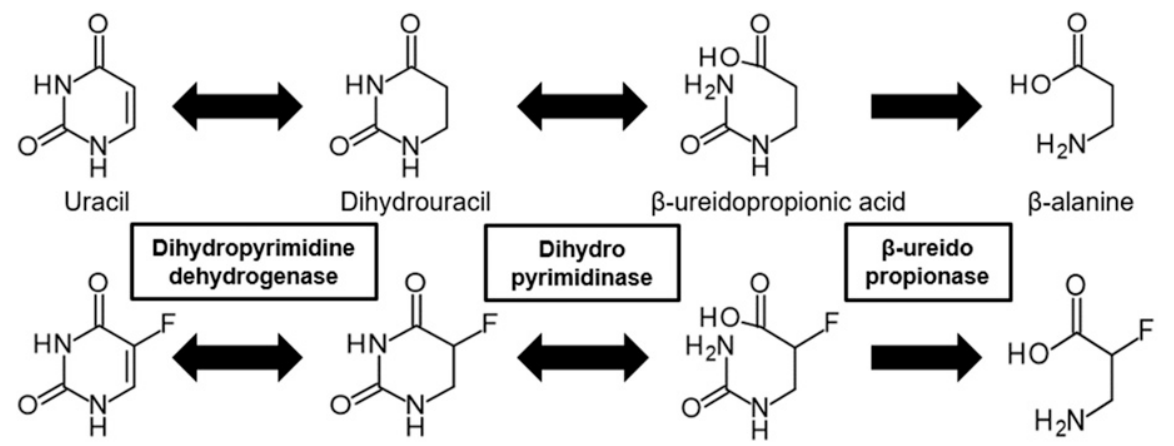

Fig. 1. Metabolic pathway of uracil and 5-FU. Uracil and 5 -FU are inactivated by dihydropyrimidine dehydrogenase, dihydropyrimidinase, and $\beta$-ureidopropionase. $\beta$-Alanine and fluoro- $\beta$-alanine are the final metabolites in this pathway.

5-Fluorouracil

Dihydro-5-fluorouracil

Fluoro- $\beta$-ureidopropionic acid

Fluoro- $\beta$-alanine

$D P Y D * 2 A)$, which results in exon 14 skipping; c. 1679T $>\mathrm{G}(D P Y D * 13$, p.I560S); c.1129-5923C $>$ G/hapB3; and c.2846A $>$ T (p.D949V) (Froehlich et al., 2015; Meulendijks et al., 2015). None of these DPYD polymorphisms has been identified in the Asian population, however (van Kuilenburg, 2004; Maekawa et al., 2007).

The Tohoku Medical Megabank Organization (ToMMo) has reported the whole-genome sequences (WGS) of 1070 healthy Japanese individuals and has constructed a Japanese population reference panel (1KJPN) (Nagasaki et al., 2015). Twenty-one DPYD allelic variants, including some novel single-nucleotide variants (SNVs), were identified in these subjects (Table 1), but the resulting functional alterations remain unknown. It is possible that rare DPYD variants could become novel genetic markers that are used to predict the adverse effects of 5-FU in the Japanese population before therapeutic administration.

In this study, we conducted in vitro assays on 21 DPD variants in 293FT cells identified in 1070 Japanese subjects under identical conditions using 5-FU as a substrate. We determined the values of kinetic parameters associated with DPD activities involved in the reduction of 5-FU and investigated the mechanism underlying the observed reduction in activity using blue-native-PAGE and threedimensional modeling. Our results help elucidate how alterations found in the amino acid sequence of DPD affect its function and support the hypothesis that rare DPYD variants could potentially serve as pharmacogenomic markers to predict severe 5-FU-related toxicity in cancer patients receiving 5-FU-based treatments in the Japanese population.

\section{Materials and Methods}

Chemicals. The following reagents were purchased from the listed sources: sodium sulfide pentahydrate $\left(\mathrm{Na}_{2} \mathrm{~S}\right)$, Wako (Osaka, Japan); 5-fluorodihydropyrimidine2,4-dione $\left(\mathrm{FUH}_{2}\right)$, Toronto Research Chemicals (North York, ON, Canada); $\beta$-nicotinamide adenine dinucleotide phosphate $(\beta$-NADPH), Oriental Yeast (Tokyo, Japan); 5-fluorouracil (5-FU), flavin adenine dinucleotide (FAD), flavin mononucleotide (FMN), ammonium iron (III) citrate, and dithiothreitol, Nacalai Tesque (Kyoto, Japan); Uracil- ${ }^{15} \mathrm{~N}_{2}$, Santa Cruz Biotechnology (Santa Cruz, CA); polyclonal anti-human DPD antibody, Millipore (Tokyo, Japan); polyclonal anti-GAPDH (glyceraldehyde 3-phosphate dehydrogenase) antibody, Sigma-Aldrich (St. Louis, MO); and horseradish peroxidase (HRP)conjugated goat anti-rabbit IgG, DakoCytomation (Glostrup, Denmark) and Santa Cruz Biotechnology. All other chemicals and reagents were of the highest quality commercially available.

DPYD Analysis by Sanger Sequencing. To confirm the sequence alterations identified by WGS, we performed Sanger sequencing analysis of DPYD by PCR amplification using peripheral blood leukocyte genomic DNA from Japanese subjects who participated in a community-based cohort study conducted by ToMMo. The local ethics committee of Tohoku University School of Medicine approved the study, and all cohort participants provided written informed consent. Genomic DNA was prepared from whole blood using a Gentra Puregene Blood Kit (Qiagen, Hilden, Germany) as previously described (Nagasaki et al., 2015).
The primer pairs used to amplify sequences containing the SNVs of the DPYD gene are listed in Table 2 . The reaction mixture contained approximately $10 \mathrm{ng}$ of genomic DNA, $0.5 \mu \mathrm{M}$ of each primer, and AmpliTaq Gold 360 Master Mix (Applied Biosystems, Foster City, CA), in a total reaction volume of $20 \mu 1$. The PCR conditions consisted of initial denaturation at $95^{\circ} \mathrm{C}$ for 10 minutes, followed by 30 cycles of denaturation at $95^{\circ} \mathrm{C}$ for 30 seconds, annealing at $60^{\circ} \mathrm{C}$ for 30 seconds, extension at $72{ }^{\circ} \mathrm{C}$ for 30 seconds, and then extension at $72^{\circ} \mathrm{C}$ for 7 minutes. The resulting amplification products were purified using the ExoSAP-IT PCR product cleanup reagent (Affymetrix, Cleveland, OH). Dyeterminator cycle sequencing was performed using the BigDye Terminator v3.1 cycle sequencing kit (Applied Biosystems). The amplicons were sequenced in both directions with an Applied Biosystems 3500xL Genetic Analyzer (Applied Biosystems)

$D P Y D$ cDNA Cloning and Construction of Expression Vectors. The plasmid containing wild-type human DPYD cDNA was constructed using TOPO cloning to insert a plasmid comprising the complete coding region of the human $D P Y D$ into the pENTR/D-TOPO vector (Thermo Fisher Scientific, Waltham, MA). The plasmid containing wild-type human DPYD cDNA was used as a template to generate $21 D P Y D$ variant constructs using a QuikChange Lightning Site-Directed Mutagenesis Kit (Agilent Technologies, Tokyo, Japan) according to the manufacturer's instructions. All the prepared constructs were confirmed by direct sequencing. Wild-type and variant $D P Y D$ cDNA fragments were subsequently subcloned into the mammalian expression vector pcDNA3.4 (Thermo Fisher Scientific).

Expression of DPD Variants in 293FT Cells. 293FT cells were cultured in Dulbecco's modified Eagle's medium (Nacalai Tesque) containing $10 \%$ fetal bovine serum at $37^{\circ} \mathrm{C}$ under $5 \% \mathrm{CO}_{2}$. Cells were plated at a density of $2.0 \times 10^{6}$ cells/100-mm dish; 24 hours after plating, cells were transfected with plasmids carrying DPYD cDNA $(7 \mu \mathrm{g})$ and lacZ $(1 \mu \mathrm{g})$ using polyethylenimine Max reagent (Polysciences Inc., Warrington, PA) according to the manufacturer's instructions. Sodium sulfide $(10 \mu \mathrm{M})$, ammonium ferric citrate $(10 \mu \mathrm{M})$, FAD $(10 \mu \mathrm{M})$, and FMN $(10 \mu \mathrm{M})$ were added at 6 hours post-transfection. After incubation for 42 hours at $37^{\circ} \mathrm{C}$, cells were scraped off, centrifuged at $1500 \mathrm{~g}$ for 5 minutes, and resuspended in a homogenization buffer containing $10 \mathrm{mM}$ Tris$\mathrm{HCl}$ (pH 7.4), $1 \mathrm{mM}$ EDTA, and 10\% glycerol. After cell homogenization, the soluble fractions were prepared by differential centrifugation at $9000 \mathrm{~g}$ for 20 minutes, followed by centrifugation of the resulting supernatant at $105,000 \mathrm{~g}$ for 60 minutes. The protein concentration was determined using a BCA protein assay kit (Thermo Fisher Scientific). The $\beta$-galactosidase activity was measured using the $\beta$-galactosidase enzyme assay system (Promega Co., Madison, WI) to allow adjustment for transfection efficiency.

Determination of DPD Protein Expression through Immunoblotting after SDS-PAGE. Soluble fractions $(10 \mu \mathrm{g})$ of 293FT cells were separated on $10 \%$ ePAGEL (ATTO, Tokyo, Japan) with an SDS buffer containing $25 \mathrm{mM}$ Tris, $192 \mathrm{mM}$ glycine, and $0.1 \%$ SDS. Immunoblotting for each DPD variant was performed in triplicate, in accordance with standard procedures. DPD was detected using a polyclonal rabbit anti-human DPD antibody (1:2000) and HRPconjugated goat anti-rabbit IgG (1:5000; DakoCytomation). The loading control used was GAPDH, which was detected using a polyclonal rabbit anti-GAPDH antibody (1:5000) and HRP-conjugated goat anti-rabbit IgG (1:10,000; Santa Cruz Biotechnology). Immunoblots were visualized using a SuperSignal West 
TABLE 1

$D P Y D$ variants identified in 1070 Japanese subjects

\begin{tabular}{|c|c|c|c|c|}
\hline dbSNP rsID & Location & $\begin{array}{l}\text { Nucleotide } \\
\text { Change }\end{array}$ & Amino Acid Substitution & Frequency $(\%)$ \\
\hline - & Exon 2 & $74 \mathrm{~A}>\mathrm{G}$ & $\mathrm{H} 25 \mathrm{R}$ & 0.05 \\
\hline- & Exon 5 & $325 \mathrm{~T}>\mathrm{A}$ & Y109N & 0.23 \\
\hline rs200562975 & Exon 5 & $451 \mathrm{~A}>\mathrm{G}$ & N151D & 0.14 \\
\hline rs 2297595 & Exon 6 & $496 \mathrm{~A}>\mathrm{G}$ & M166V & 2.24 \\
\hline rs371792178 & Exon 6 & $524 \mathrm{C}>\mathrm{T}$ & S175L & 0.05 \\
\hline- & Exon 9 & $893 \mathrm{C}>\mathrm{T}$ & $\mathrm{T} 298 \mathrm{M}$ & 0.05 \\
\hline - & Exon 9 & $937 \mathrm{G}>\mathrm{T}$ & V313L & 0.05 \\
\hline - & Exon 10 & $1003 \mathrm{G}>\mathrm{A}$ & V335M & 0.19 \\
\hline - & Exon 10 & $1097 \mathrm{G}>\mathrm{C}$ & G366A & 0.05 \\
\hline - & Exon 11 & $1139 \mathrm{C}>\mathrm{T}$ & A380V & 0.05 \\
\hline- & Exon 11 & $1150 \mathrm{~A}>\mathrm{G}$ & K384E & 0.05 \\
\hline - & Exon 11 & $1300 \mathrm{G}>\mathrm{C}$ & V434L & 0.05 \\
\hline rs 148994843 & Exon 13 & $1543 \mathrm{G}>\mathrm{A}$ & V515I & 0.05 \\
\hline rs 1801159 & Exon 13 & $1627 \mathrm{~A}>\mathrm{G}(D P Y D * 5)$ & I543V & 25.68 \\
\hline rs59086055 & Exon 14 & $1774 \mathrm{C}>\mathrm{T}$ & R592W & 0.05 \\
\hline rs 1801160 & Exon 18 & $2194 \mathrm{G}>\mathrm{A}\left(D P Y D^{*} \sigma\right)$ & V732I & 1.82 \\
\hline rs56005131 & Exon 19 & $2303 \mathrm{C}>\mathrm{A}$ & $\mathrm{T} 768 \mathrm{~K}$ & 2.20 \\
\hline- & Exon 19 & $2420 \mathrm{~A}>\mathrm{G}$ & H807R & 0.05 \\
\hline - & Exon 20 & $2476 \mathrm{G}>\mathrm{A}$ & V826M & 0.14 \\
\hline rs 188052243 & Exon 21 & $2678 \mathrm{~A}>\mathrm{G}$ & N893S & 0.19 \\
\hline- & Exon 22 & $2777 \mathrm{G}>\mathrm{T}$ & G926V & 0.05 \\
\hline
\end{tabular}

Dura Extended Duration Substrate (Thermo Fisher Scientific). Chemiluminescence was quantified using a ChemiDoc $\mathrm{XRS}^{+}$with Image Laboratory Software (Bio-Rad Laboratories, Hercules, CA).

Immunoblotting after Blue-Native PAGE. Blue-native PAGE was performed using a 5\%-20\% ePAGEL (ATTO), with running buffer containing $25 \mathrm{mM}$ Tris and $192 \mathrm{mM}$ glycine. Soluble fractions $(9 \mu \mathrm{g})$ mixed with sample buffer (62.5 mM Tris, $10 \%$ glycerol, and $0.125 \%$ G-250, $\mathrm{pH} 7.4$ ) were loaded into each gel lane in triplicate, and electrophoresis was performed at $20 \mathrm{~mA}$ for 2.5 hours at room temperature. After electrophoresis, the gels were incubated for 10 minutes with an SDS buffer (20 mM Tris, $150 \mathrm{mM}$ glycine, and $0.1 \%$ SDS, pH 7.4). Proteins were later transferred onto PVDF membranes and immunoblotted as described already. NativeMark unstained protein standard (Thermo Fisher Scientific) was used as the molecular weight marker, and proteins were detected by Coomassie Brilliant Blue staining after electrophoresis.

5-FU Reduction Assays. DPD-mediated reduction of 5-FU was measured by quantifying $\mathrm{FUH}_{2}$ using liquid chromatography-tandem mass spectrometry (LC-MS/MS). The incubation mixture consisted of the sample soluble fraction (50 $\mu \mathrm{g}$ ), $1 \mathrm{mM}$ dithiothreitol, $200 \mu \mathrm{M} \beta$-NADPH, $2.5 \mathrm{mM}$ magnesium chloride, and $50 \mathrm{mM}$ potassium phosphate buffer ( $\mathrm{pH} 7.4)$ in a total volume of $150 \mu \mathrm{l}$. After preincubation at $37^{\circ} \mathrm{C}$ for 3 minutes, reactions were initiated by adding 5-FU $(0.1$, $0.2,0.5,1,2,5,10$, or $20 \mu \mathrm{M})$. After incubating the mixtures at $37^{\circ} \mathrm{C}$ for 30 minutes, the reactions were terminated by adding $150 \mu \mathrm{l}$ of acetonitrile containing $1 \mu \mathrm{M}$ uracil- ${ }^{15} \mathrm{~N}_{2}$ as an internal standard. 5-FU reduction measurements obtained using $20 \mu \mathrm{M} 5$-FU and $50 \mu \mathrm{g}$ of the soluble fraction containing wild-type and variant DPD proteins showed that $\mathrm{FUH}_{2}$ formation was linear for incubations of up to 30 minutes. When the reaction containing $20 \mu \mathrm{M} 5$-FU was incubated for 30 minutes, the formation of $\mathrm{FUH}_{2}$ was linear in the presence of up to $50 \mu \mathrm{g}$ of soluble protein (data not shown).

After removing proteins by centrifuging reaction mixtures at $14,000 \mathrm{~g}$ for 5 minutes, $150 \mu \mathrm{l}$ of the supernatant was vacuum-dried at $40^{\circ} \mathrm{C}$ for 1 hour and dissolved in $75 \mu 1$ of $0.1 \%$ (v/v) formic acid in water. Subsequently, $5 \mu 1$ of the solution was injected into an LC-MS/MS system, and $\mathrm{FUH}_{2}$ was measured using the system in the positive ion-detection mode on the electrospray ionization interface (API 5000 triple-quadrupole mass spectrometer; SCIEX, Framingham, MA). High-performance liquid chromatography separation was performed using a Prominence HPLC system (SHIMADZU, Kyoto, Japan), and a CAPCELL PAK ADME S3 column $(2.1 \times 150 \mathrm{~mm}, 3.0-\mu \mathrm{m}$ particle size; OSAKA SODA, Osaka, Japan) maintained at $8^{\circ} \mathrm{C}$. $\mathrm{FUH}_{2}$ was eluted isocratically at a flow rate of $200 \mu \mathrm{l} / \mathrm{min}$ using a mobile phase consisting of $0.1 \%(\mathrm{v} / \mathrm{v})$ formic acid in water. The LC-MS/MS system was controlled by the Analyst 1.5 software (Sciex), which was also used to analyze the data. The standard curve for $\mathrm{FUH}_{2}$ was constructed in the $0.025-10 \mu \mathrm{M}$ range using authentic metabolite standards. The enzymatic activity was normalized to the corresponding DPD expression level, adjusted for transfection efficiency.

TABLE 2

Polymerase chain reaction primers used to amplify sequences of the human DPYD gene

\begin{tabular}{|c|c|c|c|}
\hline \multirow{2}{*}{ Nucleotide Change } & \multicolumn{2}{|c|}{ Primer $\left(5^{\prime}-3^{\prime}\right)$} & \multirow{2}{*}{ Product Length (bp) } \\
\hline & Sense & Antisense & \\
\hline $74 \mathrm{~A}>\mathrm{G}$ & gacaagtgagagagaccgtgtctc & tcttgccttacaatgtgtggagtg & 287 \\
\hline $325 \mathrm{~T}>\mathrm{A} 451 \mathrm{~A}>\mathrm{G}$ & gatggttcctgatagtattgaaacc & gctgtgtgtcacactaaaatgttg & 433 \\
\hline $496 \mathrm{~A}>\mathrm{G} 524 \mathrm{C}>\mathrm{T}$ & agaaaggaaagactgaaagttagcc & gtaggcattaccttaaccaccaac & 402 \\
\hline $893 \mathrm{C}>\mathrm{T} 937 \mathrm{G}>\mathrm{T}$ & atctaataggaaaagccctcctc & ttacatttgggtcttaggcaagg & 294 \\
\hline $1003 \mathrm{G}>\mathrm{A} 1097 \mathrm{G}>\mathrm{C}$ & caagtatctttgagctgtcatgcag & acactaacaagaagcccttgagtattg & 483 \\
\hline $1139 \mathrm{C}>\mathrm{T} 1150 \mathrm{~A}>\mathrm{G} 1300 \mathrm{G}>\mathrm{C}$ & actgaactcaggtttggtgaaag & ccctgaaagctagaaactattacagc & 430 \\
\hline $1543 \mathrm{G}>\mathrm{A} \quad 1627 \mathrm{~A}>\mathrm{G}$ & aattcggatgctgtgttgagtg & aaccccatccagcttcaaaag & 280 \\
\hline $1774 \mathrm{C}>\mathrm{T}$ & tcctctgcaaaaatgtgagaagg & cagcctttagttcagtgacactttg & 271 \\
\hline $2194 \mathrm{G}>\mathrm{A}$ & tgaatgggttttaactatcgtgtc & aagtgggcaacacctaccag & 220 \\
\hline $2303 \mathrm{C}>\mathrm{A} \quad 2420 \mathrm{~A}>\mathrm{G}$ & gaaatttgtccgtgcgetgtc & atcccaaatggectccttttc & 372 \\
\hline $2476 \mathrm{G}>\mathrm{A}$ & catcatgcctcaaacagtgcc & gaaaccaaggctgagttctcaag & 351 \\
\hline $2678 \mathrm{~A}>\mathrm{G}$ & gaaacaatccctagacacacatttg & catgcttgccagtgttctaaaaag & 476 \\
\hline $2777 \mathrm{G}>\mathrm{T}$ & aggcatgcatattgcccatc & ttccagcaggattcttacetgg & 365 \\
\hline
\end{tabular}


A

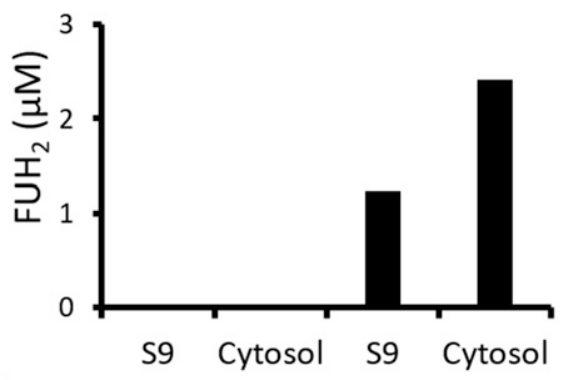

B
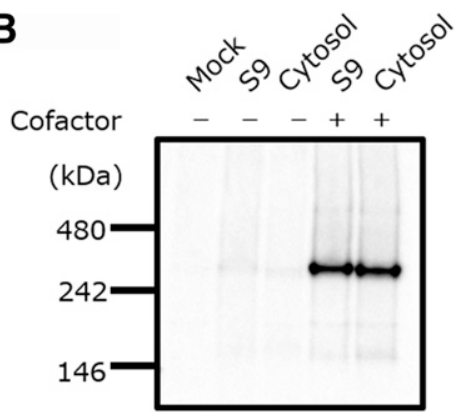

Fig. 2. Effect of DPD cofactors (sodium sulfide, ammonium ferric citrate, FAD, and FMN) on enzymatic activity. (A) DPD activity with or without the four cofactors was determined at $50 \mu \mathrm{M}$ 5-FU. (B) DPD proteins in 293FT cells with or without the four cofactors were determined through blue-native PAGE and immunoblotting analysis.

Cofactor

Data Analysis. The kinetic data were analyzed using the Enzyme Kinetics Module of SigmaPlot 12.5 (Systat Software, Inc., Chicago, IL), a curvefitting program based on nonlinear regression analysis. The values of the Michaelis constant $\left(K_{m}\right)$, maximum velocity $\left(V_{\max }\right)$, and intrinsic clearance $\left(C L_{i n t}=V_{\max } / K_{m}\right)$ were determined using the software. $V_{\max }$ was calculated using the average DPD expression-level values in triplicate. All values are expressed herein as means \pm S.D. of experiments performed in triplicate. Statistical analyses of the protein expression levels and kinetic parameters were performed by analysis of variance using Dunnett's T3 test or the Kruskal-Wallis method (IBM SPSS Statistics Version 22; International Business Machines, Armonk, NY). Differences or correlations with $P<0.05$ were considered significant.
A

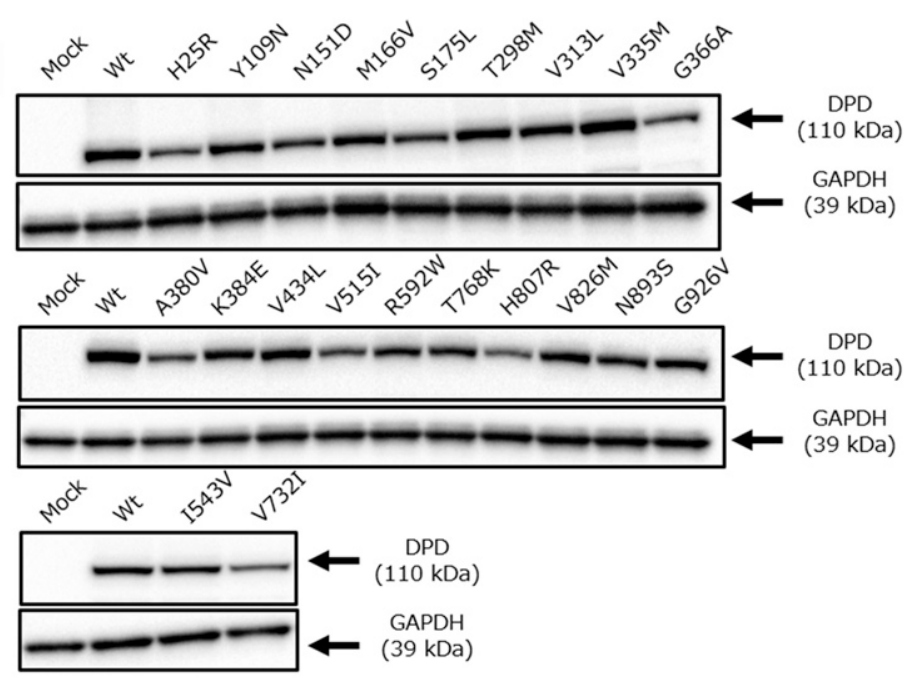

B

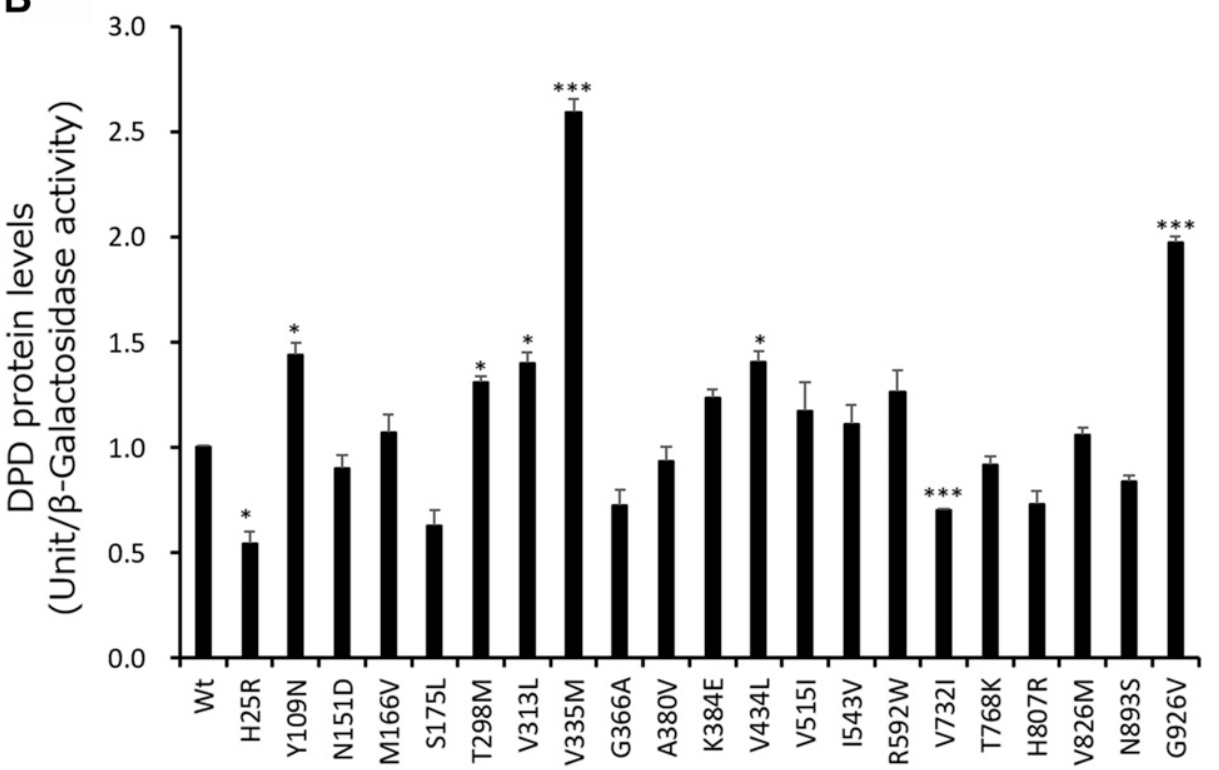

Fig. 3. Expression levels of wild-type and variant DPD proteins. (A) DPD protein levels were determined through SDS-PAGE and immunoblotting analysis. (B) DPD expression levels were normalized relative to $\beta$-galactosidase activity in 293FT cells. Bars represent means \pm S.D. of three independent assays. $* P<0.05 ; * * * P<0.005$ compared with wild-type DPD. 
Three-Dimensional Simulation Modeling Analysis. A homology model of human DPD was generated by SWISS-MODEL (https://swissmodel.expasy.org/), based on the crystal structure of pig DPD (RSCB Protein Data Bank, accession code 1H7W). The CDOCKER protocol for Discovery Studio 2.5 (Accelrys, San Diego, CA) was used to create docked DPD-5-FU structures. The Discovery Studio 4.5 visualizer was used for the three-dimensional imaging of DPD.

\section{Results}

To confirm the sequence alterations identified by WGS, we performed Sanger sequencing analysis of the DPYD. For exon SNVs, the WGS results were the same as those from the Sanger sequencing. Twenty-one DPYD allelic variants, including some novel SNVs, were identified in the Japanese subjects.

To analyze the effects of sodium sulfide, ammonium ferric citrate, FAD, and FMN on DPD activity expressed in 293FT cells, we cultured 293FT cells with or without these cofactors after transfection and measured DPD activity. The production of $\mathrm{FUH}_{2}$ by DPD expressed with the four cofactors was observed using $50 \mu \mathrm{M}$ 5-FU, whereas the production of $\mathrm{FUH}_{2}$ of DPD, expressed without cofactors, could not be observed under the same conditions (Fig. 2A). Furthermore, bands were detected in immunoblots after blue-native PAGE only when DPD was expressed using the four cofactors (Fig. 2B).

To functionally characterize $D P Y D$ variants, wild-type DPD and 21 DPD variant proteins were transiently expressed in 293FT cells. The expression levels of these DPD proteins were determined by performing quantitative immunoblotting after SDS-PAGE with a polyclonal DPD antibody, which recognized all DPD variants expressed in 293FT cells (Fig. 3A). GAPDH, used as a loading control, was stained at approximately the same level in the soluble fractions of all transfected 293FT cells. Endogenous DPD protein was not detected in 293FT cells transfected with an empty vector (mock). The average levels of wildtype and variant DPD proteins, adjusted for transfection efficiency using the previously measured $\beta$-galactosidase activity, are shown in Fig. 3B. We defined DPD protein levels equal in intensity to $10 \mu \mathrm{g}$ of wild-type DPD as 1 DPD unit. Relative to wild-type, the DPD unit levels for Y109N, T298M, V313L, and V434L were slightly elevated $(P<0.05)$, and those for V335M and G926V were considerably elevated $(P<0.005)$; those for H25R were slightly lower $(P<0.05)$, and those for V732I were considerably lower $(P<0.005)$. The levels of the remaining 13 variants did not differ significantly from that of wild-type DPD.

We determined the kinetic parameters for the reduction of 5-FU by wild-type DPD and the 21 DPD variants (Table 3). The nonlinear regression curves for the Michaelis-Menten kinetics are shown in Fig. 4. The $K_{m}, V_{\text {max }}$, and $C L_{\text {int }}$ values for the reduction of 5-FU by wild-type DPD were $2.37 \mu \mathrm{M}, 5.53 \mathrm{pmol}^{\circ} \mathrm{min}^{-1} \cdot \mathrm{U}^{-1}$, and 2.34 $\mu \mathrm{l} \bullet \mathrm{min}^{-1} \bullet \mathrm{U}^{-1}$, respectively. For G926V, the kinetic parameters could not be determined because no 5-FU reduction activity was detected. Relative to wild-type DPD, 12 variants (T298M, V313L, G366A, A380V, K384E, V434L, V515I, R592W, T768K, H807R, V826M, and N893S) exhibited significantly lower $V_{\max }$ values $(P<0.05)$, and 10 variants (T298M, V313L, V335M, A380V, V434L, V515I, R592W, T768K, H807R, and V826M) exhibited significantly lower $C L_{\text {int }}$ values $(P<0.05)$.

Immunoblotting after blue-native PAGE under non-SDS conditions revealed approximately $242-\mathrm{kDa}$ bands, estimated as dimeric forms of wild-type DPD (Fig. 5). Identical bands of wild-type DPD were detected for H25R, Y109N, M166V, S175L, T298M, V335M, K384E, V434L, I543V, and V732I. Compared with wild-type DPD, the intensity of the dimeric forms of 10 DPD variants (N151D, V313L, G366A, A380V, V515I, R592W, T768K, H807R, V826M, and N893S) was decreased. No dimeric band was detected for the G926V DPD variant.
TABLE 3

Kinetic parameters of dihydropyrimidine dehydrogenase (DPD) variants for 5-fluorouracil metabolism

These data represent the mean \pm S.D. of three independently performed catalytic assays. The kinetic parameters of G926V could not be determined because the enzymatic activity was not detected at the highest substrate concentration assayed ( $20 \mu \mathrm{M} 5$-fluorouracil).

\begin{tabular}{|c|c|c|c|c|}
\hline Variants & $K_{m}$ & $V_{\max }$ & $C L_{\text {int }}\left(V_{\max } / K_{m}\right)$ & Wild-Type $C L_{\text {int }}$ \\
\hline & $\mu M$ & $\mathrm{pmol} / \mathrm{min}$ per unit & $\mu \mathrm{l} / \mathrm{min}$ per unit & $\%$ \\
\hline Wild-type & $2.37 \pm 0.24$ & $5.53 \pm 0.40$ & $2.34 \pm 0.15$ & 100.0 \\
\hline $\mathrm{H} 25 \mathrm{R}$ & $1.67 \pm 0.13$ & $6.07 \pm 0.29$ & $3.64 \pm 0.16^{*}$ & 155.9 \\
\hline Y109N & $1.97 \pm 0.05$ & $3.63 \pm 0.09$ & $1.84 \pm 0.06$ & 78.9 \\
\hline N151D & $1.44 \pm 0.26$ & $3.50 \pm 0.32$ & $2.50 \pm 0.59$ & 107.1 \\
\hline M166V & $2.59 \pm 0.26$ & $4.65 \pm 0.11$ & $1.81 \pm 0.14$ & 77.3 \\
\hline S175L & $1.95 \pm 0.34$ & $5.89 \pm 0.40$ & $3.05 \pm 0.32$ & 130.6 \\
\hline $\mathrm{T} 298 \mathrm{M}$ & $1.80 \pm 0.06$ & $2.10 \pm 0.08^{*}$ & $1.17 \pm 0.03^{*}$ & 50.0 \\
\hline V313L & $1.32 \pm 0.15$ & $0.92 \pm 0.01 *$ & $0.70 \pm 0.08 * *$ & 30.1 \\
\hline V335M & $2.92 \pm 0.17$ & $3.23 \pm 0.19$ & $1.11 \pm 0.13^{*}$ & 47.4 \\
\hline G366A & $1.30 \pm 0.08$ & $2.14 \pm 0.01 *$ & $1.65 \pm 0.09$ & 70.5 \\
\hline A380V & $0.88 \pm 0.09$ & $0.69 \pm 0.03^{*}$ & $0.78 \pm 0.05 *$ & 33.3 \\
\hline $\mathrm{K} 384 \mathrm{E}$ & $1.57 \pm 0.08$ & $2.52 \pm 0.14^{*}$ & $1.60 \pm 0.05$ & 68.4 \\
\hline V434L & $2.13 \pm 0.12$ & $2.16 \pm 0.05^{*}$ & $1.02 \pm 0.08^{*}$ & 43.6 \\
\hline V515I & $1.25 \pm 0.08$ & $1.05 \pm 0.04 *$ & $0.83 \pm 0.03 *$ & 35.5 \\
\hline I543V & $2.01 \pm 0.18$ & $4.79 \pm 0.20$ & $2.38 \pm 0.18$ & 101.8 \\
\hline R592W & $3.50 \pm 0.43$ & $0.14 \pm 0.01 *$ & $0.04 \pm 0.00 *$ & 1.7 \\
\hline V732I & $2.85 \pm 0.46$ & $7.51 \pm 0.45$ & $2.67 \pm 0.35$ & 114.1 \\
\hline $\mathrm{T} 768 \mathrm{~K}$ & $1.46 \pm 0.07$ & $1.64 \pm 0.06^{*}$ & $1.12 \pm 0.02 *$ & 47.9 \\
\hline H807R & $1.10 \pm 0.04$ & $1.30 \pm 0.07 *$ & $1.18 \pm 0.09 *$ & 50.4 \\
\hline V826M & $1.22 \pm 0.12$ & $1.00 \pm 0.08^{*}$ & $0.82 \pm 0.03^{*}$ & 35.0 \\
\hline N893S & $1.92 \pm 0.28$ & $2.79 \pm 0.15^{*}$ & $1.47 \pm 0.19$ & 62.8 \\
\hline
\end{tabular}

$* P<0.05 ; * * P<0.01$ compared with wild-type DPD

\section{Discussion}

DPD deficiency can result in severe toxicity during 5-FU-based cancer treatment (Ezzeldin and Diasio, 2004; Mounier-Boutoille et al., 2010; Sahu et al., 2016). Twenty-one DPYD allelic variants were identified in 1070 Japanese individuals, but the functional alterations caused by these variants remain unknown. In this study, we characterized the enzymatic activity of wild-type DPD and 21 DPD variants using recombinant proteins expressed in 293FT cells and determined the kinetic parameters of these variant enzymes associated with 5-FU reduction activity. We also investigated the mechanism underlying the reduced activity observed for these variants using blue-native PAGE and three-dimensional modeling-based structural analysis.

The effects of sodium sulfide, ammonium ferric citrate, FAD, and FMN on DPD activity were analyzed using cultured 293FT cells with or without post-transfection treatment with these cofactors. Enzymatic activity and an approximately $242-\mathrm{kDa}$ band, estimated as DPD dimeric forms, were observed only in DPD variants expressed with the four cofactors. These results suggest that DPD enzymatic activity is dimerization-dependent and, consequently, that the four cofactors play a critical role in 5-FU reduction.

To characterize and assess the functional effects of the 21 DPYD mutations on DPD activity in the Japanese population, wild-type and 21 variant DPD proteins were transiently expressed in 293FT cells with the four cofactors. The kinetic parameters for 5-FU reduction activity were determined for wild-type and 20 DPD variants expressed in 293FT cells, with the exclusion of G926V. Ten DPD variants (T298M, V313L, V335M, A380V, V434L, V515I, R592W, T768K, H807R, and V826M) exhibited significantly reduced $C L_{\text {int }}$ values relative to wild-type DPD. Conversely, the metabolic activities of 5-FU were almost eliminated in G926V. Offer et al. (2014) reported that of six variants (N151D, M166V, V515I, R592W, T768K, and N893S) expressed in HEK293T/c17 cells using 5-FU as a substrate, only N151D, M166V, V515I, and T768K exhibited activity equal to that of wild-type DPD. In contrast, R592W and N893S exhibited 

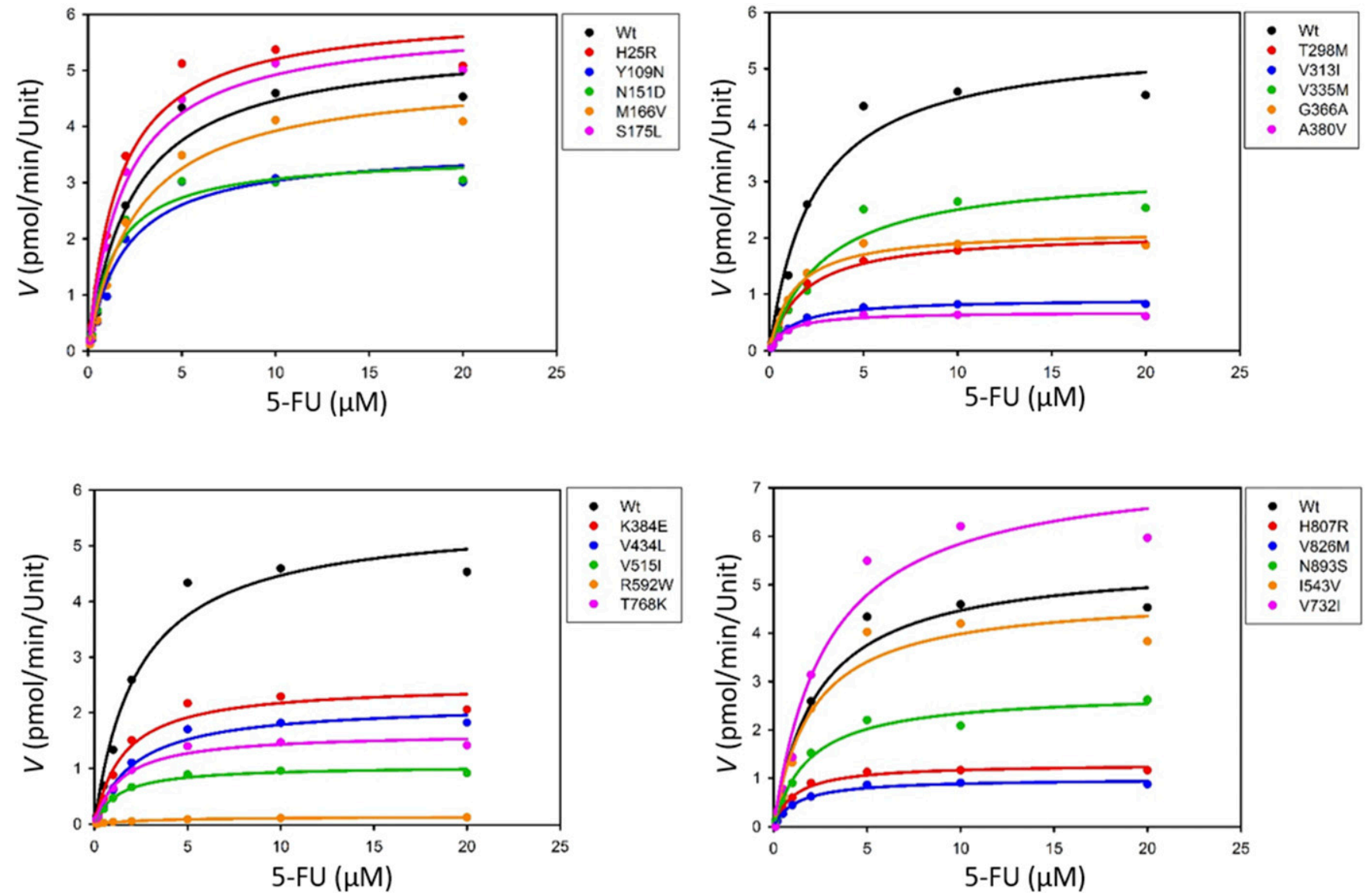

\begin{tabular}{ll|} 
- & $\mathrm{Wt}$ \\
- $\mathrm{H} 807 \mathrm{R}$ \\
- $\mathrm{V} 826 \mathrm{M}$ \\
- \\
- $1593 \mathrm{~S}$ \\
- & $\mathrm{V} 732 \mathrm{~V}$ \\
\hline
\end{tabular}

Fig. 4. Michaelis-Menten curves of DPD variants. The kinetic parameters $K_{m}, V_{\max }$, and intrinsic clearance of 5-FU reduction were determined.

reduced activity. The enzymatic activities of N151D, M166V, R592W, and N893S measured using 293FT cells were similar to those previously reported, although the $C L_{i n t}$ values obtained for V515I and $\mathrm{T} 768 \mathrm{~K}$ were significantly reduced compared with wild-type DPD. This discrepancy might be due to differences in measurement conditions, substrate concentrations, or expression systems.
Collectively, these data indicate that reduction in DPD enzymatic activity might induce accumulation of 5-FU in carriers of inactive variant enzymes during 5-FU-based treatment administration. Variants exhibiting altered activity could be responsible for interindividual differences observed in pyrimidine metabolism and the appearance of severe adverse effects in cancer chemotherapy.
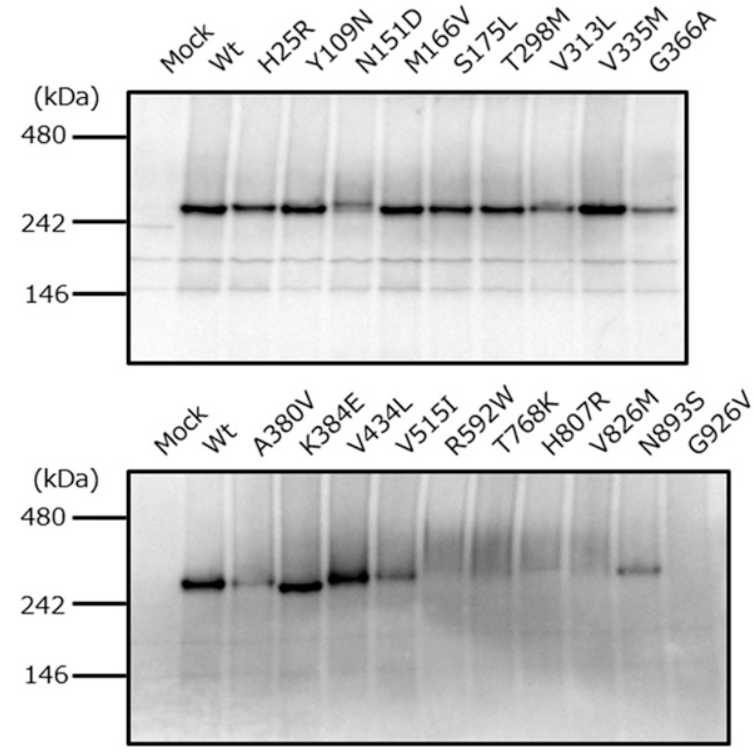

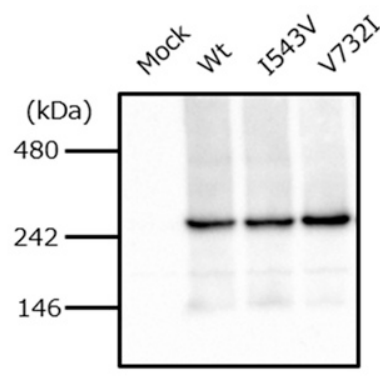

Fig. 5. Blue-native PAGE and immunoblotting analysis showing immunoreactive DPD variant proteins. Blue-native PAGE was performed using tris-glycine buffer and 5\%-20\% polyacrylamide gels; $9 \mu \mathrm{g}$ of soluble fractions of DPD variant proteins was loaded into each lane in triplicate. DPD variants were detected using polyclonal antibodies against human DPD. 

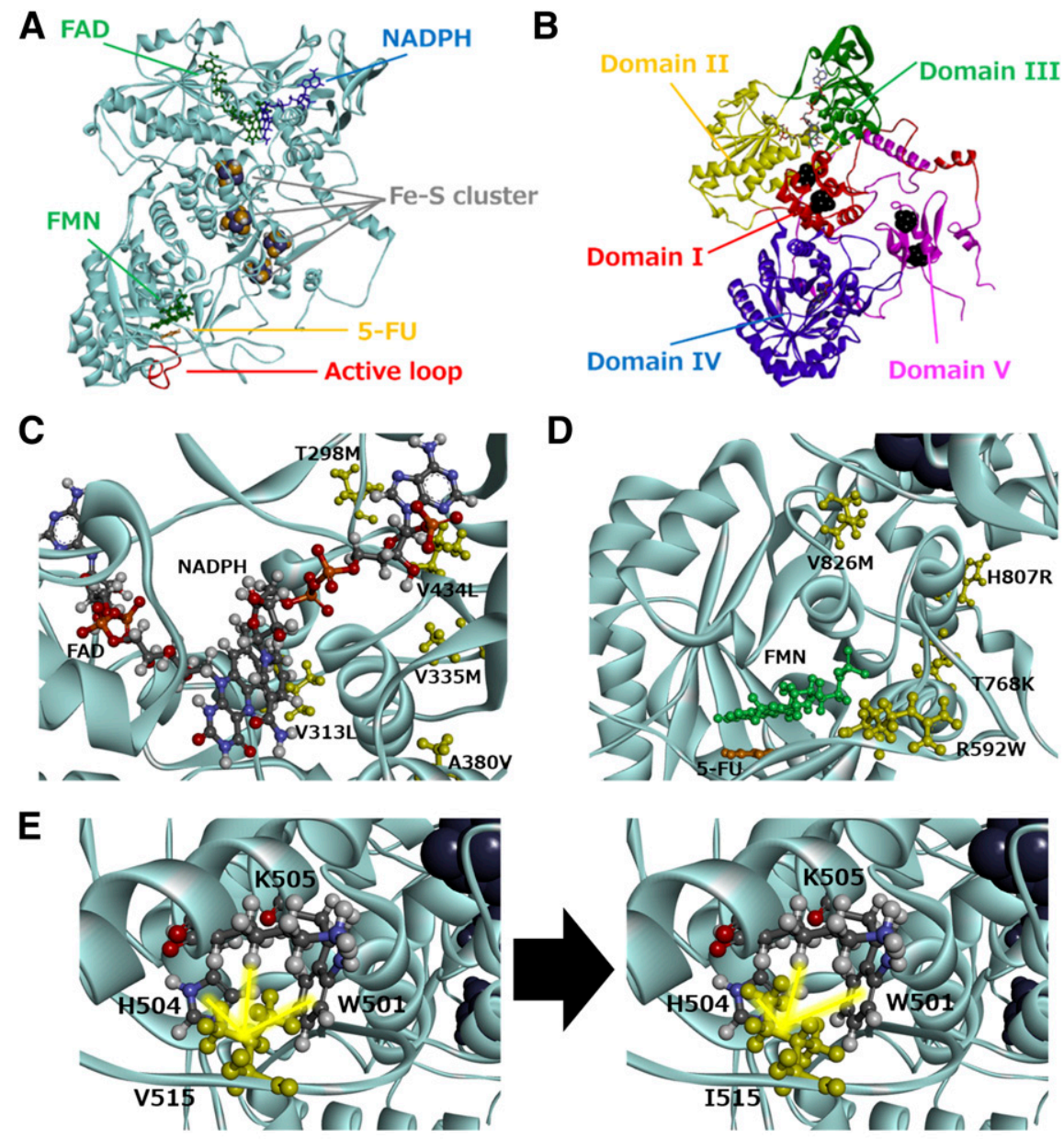

Fig. 6. DPD structural analysis. (A) Diagram showing the overall structure of human DPD, FAD, NADPH, FMN, and FeS clusters. 5-FU is colored yellow, FAD and FMN are colored green, NADPH is colored blue, and the Fe-S clusters are shown as gray spheres. (B) The DPD subunit. The distinct domains are colored differently. (C) Amino acid substitutions for five variants (T298M, V313L, V335M, A380V, and V434L) within the FAD binding site. (D) Amino acid substitutions of four variants (R592W, T768K, H807R, and V826M) within the substrate-binding site. (E) Diagrams showing a fragment of the crystal structures of wild-type DPD (left panel) and V515I (right panel). The V515 and I515 residues are shown in yellow. (F) The diagram of a fragment of the crystal structure of DPD wild-type (left panel) and G926V (right panel). G926 and V926 are shown in yellow.

$\mathbf{F}$

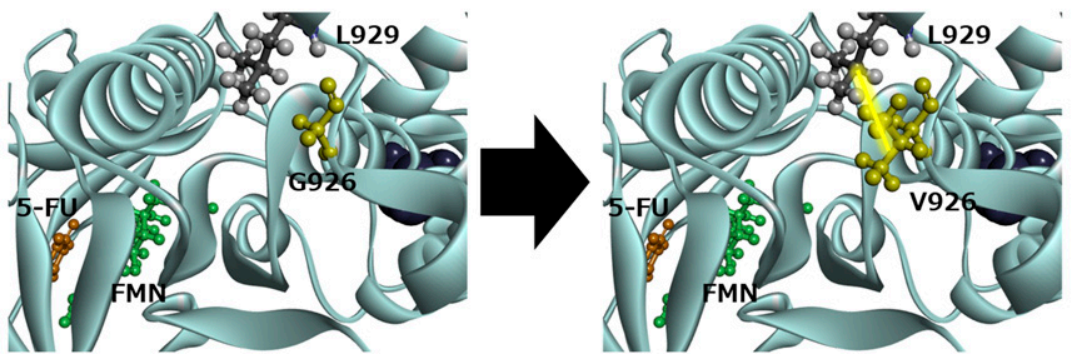

The structure of human DPD exhibited a native enzyme composed of identical dimer subunits containing one FMN, one FAD, and four FeS clusters (Lu et al., 1992; Dobritzsch et al., 2001, 2002; Mattison et al., 2002; Schnackerz et al., 2004). To determine the relationship between enzymatic activity and subunit dimerization, we performed immunoblotting after blue-native PAGE, which revealed 242-kDa DPD dimeric forms for all variants except the G926V variant. In contrast, no band was observed for the inactive G926V variant. Our data suggest that DPD dimerization plays a critical role in DPD-dependent 5-FU reduction.

Amino acid substitutions through nonsynonymous DPYD mutations might cause conformational changes in DPD active site or dimerization interaction site, and thus DPD activity could be suppressed through dimerization inhibition. Thus, we used three-dimensional modeling to analyze the effect of amino acid substitutions on DPD conformation. The human DPD structure was generated by homology modeling, based on the crystal structure for pig DPD. The DPD protein consists of five major domains (Fig. 6, A and B) (Dobritzsch et al., 2001, 2002; Mattison et al., 2002). Domain I (residues 27-172) contains two of the FeS clusters and has an exclusively $\alpha$-helical secondary structure. The FAD- and NADPH-binding domains II (residues 173-286, 442-524) and III (residues 287-441) both contain a central parallel $\beta$-sheet surrounded by $\alpha$-helices, forming a Rossman-type nucleotide binding motif. Domain IV, which binds to FMN and the substrate, belongs to the glycolate oxidase family of flavoproteins, with the typical $(\beta / \alpha)_{8}$-barrel fold. Domain V (residues 1-26, 848-1025) contains two FeS clusters. Within the dimer, the DPD dimer redox cofactors are organized in two electron transfer chains connecting the binding sites for NADPH and the substrate (Lohkamp et al., 2010). T298M, V313L, V335M, A380V, and V434L were located on the FAD- and NADPH-binding sites (Fig. 6C). These amino acid substitutions might prevent binding of NADPH, resulting in considerably decreased activity. In contrast, the R592W, T768K, H807R, and V826M substitutions affected the regions near the FMN and substrate-binding domains (Fig. 6D), which caused an active site conformational change. Closeup views of the V515I and G926V mutation sites are shown in Fig. 6, E and F, respectively. V515 participates in a hydrophobic interaction with W501, H504, and K505 
near the FAD binding site and FeS clusters. The 1515 mutation decreases activity by affecting the binding ability or electron transfer of FAD. G926 is located on domain V, near the active site. V926 substitution results in a hydrophobic interaction with L929 and inhibits dimerization, which results in diminished enzymatic activity. Our data provide evidence that the conformations of these domains and the active site might play a crucial role in DPD activity.

In conclusion, wild-type and 21 variant DPD proteins were expressed in 293FT cells, and their enzymatic activities were characterized in vitro. Eleven variants tested in this study exhibited significantly decreased enzymatic activity because of dimerization inhibition or conformational changes in each domain. These variants could contribute to the significant interindividual variability observed in the pharmacokinetics and pharmacodynamics of 5-FU and its oral prodrugs. Notably, rare $D P Y D$ variants could potentially serve as novel pharmacogenomic markers associated with severe 5-FU toxicity in Japanese and Asian patients. These findings provide insights that could facilitate further genotype-phenotype correlation studies of interindividual differences in drug efficacy and toxicity arising from disparities in DPD activity.

\section{Acknowledgments}

We thank the Biomedical Research Core of Tohoku University Graduate School of Medicine for technical support and Evelyn Marie Gutierrez Rico, Tohoku University, for proofreading the manuscript.

\section{Authorship Contributions}

Participated in the research design: Hishinuma, Narita, Akai, Hiratsuka. Conducted experiments: Hishinuma, Narita, Saito, Akai, Nakanishi.

Contributed new reagents or analytic tools: Saito, Maekawa, Nagasaki, Yamamoto, Yamaguchi, Mano, Hirasawa, Hiratsuka.

Performed data analysis: Hishinuma, Narita, Saito, Maekawa, Akai, Yasuda, Nagasaki, Yamamoto, Hiratsuka.

Wrote or contributed to the writing of the manuscript: Hishinuma, Hiratsuka.

\section{References}

Al-Sanna'a NA, Van Kuilenburg AB, Atrak TM, Abdul-Jabbar MA, and Van Gennip AH (2005) Dihydropyrimidine dehydrogenase deficiency presenting at birth. J Inherit Metab Dis $\mathbf{2 8}$ $793-796$.

Daher GC, Harris BE, and Diasio RB (1990) Metabolism of pyrimidine analogues and their nucleosides. Pharmacol Ther 48:189-222.

Dobritzsch D, Ricagno S, Schneider G, Schnackerz KD, and Lindqvist Y (2002) Crystal structure of the productive ternary complex of dihydropyrimidine dehydrogenase with NADPH and 5-iodouracil. Implications for mechanism of inhibition and electron transfer. J Biol Chem 277: 13155-13166.

Dobritzsch D, Schneider G, Schnackerz KD, and Lindqvist Y (2001) Crystal structure of dihydropyrimidine dehydrogenase, a major determinant of the pharmacokinetics of the anti-cance drug 5-fluorouracil. EMBO J 20:650-660.

Ezzeldin H and Diasio R (2004) Dihydropyrimidine dehydrogenase deficiency, a pharmacogenetic syndrome associated with potentially life-threatening toxicity following 5-fluorouracil administration. Clin Colorectal Cancer 4:181-189.

Froehlich TK, Amstutz U, Aebi S, Joerger M, and Largiadèr CR (2015) Clinical importance of risk variants in the dihydropyrimidine dehydrogenase gene for the prediction of early-onset fluoropyrimidine toxicity. Int J Cancer 136:730-739.

Ide H, Kikuchi E, Hasegawa M, Hattori S, Yasumizu Y, Miyajima A, and Oya M (2013) Therapeutic enhancement of S-1 with CPT-11 through down-regulation of thymidylate synthase in bladder cancer. Cancer Med 2:488-495.
Kim JY, Shin E, Kim JW, Lee HS, Lee DW, Kim SH, Lee JO, Kim YJ, Kim JH, Bang SM, et al. (2015) Impact of intratumoral expression levels of fluoropyrimidine-metabolizing enzymes on treatment outcomes of adjuvant S-1 therapy in gastric cancer. PLoS One 10:e120324.

Kunicka T, Prochazka P, Krus I, Bendova P, Protivova M, Susova S, Hlavac V, Liska V, Novak P, Schneiderova M, et al. (2016) Molecular profile of 5-fluorouracil pathway genes in colorectal carcinoma. BMC Cancer 16:795.

Lohkamp B, Voevodskaya N, Lindqvist Y, and Dobritzsch D (2010) Insights into the mechanism of dihydropyrimidine dehydrogenase from site-directed mutagenesis targeting the active site loop and redox cofactor coordination. Biochim Biophys Acta 1804:2198-2206.

Lu ZH, Zhang R, and Diasio RB (1992) Purification and characterization of dihydropyrimidine dehydrogenase from human liver. $J$ Biol Chem 267:17102-17109.

Maekawa K, Saeki M, Saito Y, Ozawa S, Kurose K, Kaniwa N, Kawamoto M, Kamatani N, Kato $\mathrm{K}$, Hamaguchi T, et al. (2007) Genetic variations and haplotype structures of the DPYD gene encoding dihydropyrimidine dehydrogenase in Japanese and their ethnic differences. $J$ Hum Genet 52:804-819.

Mattison LK, Johnson MR, and Diasio RB (2002) A comparative analysis of translated dihydropyrimidine dehydrogenase cDNA; conservation of functional domains and relevance to genetic polymorphisms. Pharmacogenetics 12:133-144.

Meulendijks D, Henricks LM, Sonke GS, Deenen MJ, Froehlich TK, Amstutz U, Largiadèr CR, Jennings BA, Marinaki AM, Sanderson JD, et al. (2015) Clinical relevance of DPYD variants c. $1679 \mathrm{~T}>\mathrm{G}, \mathrm{c} .1236 \mathrm{G}>\mathrm{A} / \mathrm{HapB} 3$, and $\mathrm{c} .1601 \mathrm{G}>\mathrm{A}$ as predictors of severe fluoropyrimidineassociated toxicity: a systematic review and meta-analysis of individual patient data. Lancet Oncol 16:1639-1650.

Mounier-Boutoille H, Boisdron-Celle M, Cauchin E, Galmiche JP, Morel A, Gamelin E, and Matysiak-Budnik T (2010) Lethal outcome of 5-fluorouracil infusion in a patient with a total DPD deficiency and a double DPYD and UTG1A1 gene mutation. Br J Clin Pharmacol 70: 280-283.

Nagasaki M, Yasuda J, Katsuoka F, Nariai N, Kojima K, Kawai Y, Yamaguchi-Kabata Y, Yokozawa J, Danjoh I, Saito S, et al.; ToMMo Japanese Reference Panel Project (2015) Rare variant discovery by deep whole-genome sequencing of 1,070 Japanese individuals. Nat Commun 6:8018

Offer SM, Fossum CC, Wegner NJ, Stuflesser AJ, Butterfield GL, and Diasio RB (2014) Comparative functional analysis of DPYD variants of potential clinical relevance to dihydropyrimidine dehydrogenase activity. Cancer Res 74:2545-2554.

Okuma Y, Hosomi Y, Miyamoto S, Shibuya M, Okamura T, and Hishima T (2016) Correlation between S-1 treatment outcome and expression of biomarkers for refractory thymic carcinoma [published correction appears in BMC Cancer (2016) 16:272]. BMC Cancer 16:156.

Rothenberg ML, Meropol NJ, Poplin EA, Van Cutsem E, and Wadler S (2001) Mortality associated with irinotecan plus bolus fluorouracil/leucovorin: summary findings of an independent panel. J Clin Oncol 19:3801-3807.

Sahu A, Ramaswamy A, and Ostwal V (2016) Dihydro pyrimidine dehydrogenase deficiency in patients treated with capecitabine based regimens: a tertiary care centre experience. J Gastrointest Oncol 7:380-386.

Saltz LB, Niedzwiecki D, Hollis D, Goldberg RM, Hantel A, Thomas JP, Fields AL, and Mayer RJ (2007) Irinotecan fluorouracil plus leucovorin is not superior to fluorouracil plus leucovorin alone as adjuvant treatment for stage III colon cancer: results of CALGB 89803. J Clin Oncol 25:3456-3461.

Schnackerz KD, Dobritzsch D, Lindqvist Y, and Cook PF (2004) Dihydropyrimidine dehydrogenase: a flavoprotein with four iron-sulfur clusters. Biochim Biophys Acta 1701:61-74.

Sistonen J, Smith C, Fu Y-K, and Largiadèr CR (2012) A new DPYD genotyping assay for improving the safety of 5-fluorouracil therapy. Clin Chim Acta 414:109-111.

Thomas F, Hennebelle I, Delmas C, Lochon I, Dhelens C, Garnier Tixidre C, Bonadona A, Pene N, Goncalves A, Delord JP, et al. (2016) Genotyping of a family with a novel deleterious DPYD mutation supports the pretherapeutic screening of DPD deficiency with dihydrouracil/uracil ratio. Clin Pharmacol Ther 99:235-242.

Twelves C, Wong A, Nowacki MP, Abt M, Burris H, III, Carrato A, Cassidy J, Cervantes A, Fagerberg J, Georgoulias V, et al. (2005) Capecitabine as adjuvant treatment for stage III colon cancer. $N$ Engl J Med 352:2696-2704.

van Kuilenburg AB (2004) Dihydropyrimidine dehydrogenase and the efficacy and toxicity of 5-fluorouracil. Eur J Cancer 40:939-950.

van Kuilenburg AB, Meijer J, Maurer D, Dobritzsch D, Meinsma R, Los M, Knegt LC, Zoetekouw L, Jansen RL, Dezentjé V, et al. (2017) Severe fluoropyrimidine toxicity due to novel and rare DPYD missense mutations, deletion and genomic amplification affecting DPD activity and mRNA splicing. Biochim Biophys Acta 1863:721-730.

Vaudo CE, Gil B, Galuski K, Zarwan C, and Nugent FW (2016) Early-Onset 5-fluorouracil toxicity in a patient negative for dihydropyrimidine dehydrogenase mutations: the clinical course of reversal with uridine triacetate. Pharmacotherapy 36:e178-e182.

Address correspondence to: Dr. Masahiro Hiratsuka, Laboratory of Pharmacotherapy of Life-Style Related Diseases, Graduate School of Pharmaceutical Sciences, Tohoku University, Sendai, Japan, 6-3, Aoba, Aramaki, Aoba-ku, Sendai 980-8578, Japan. E-mail: mhira@m.tohoku.ac.jp 